\title{
Circadian variation of pain as a measure of the analgesia requirements during the first 24-postoperative hours in patients using an opioid Patient Controlled Analgesia delivery system
}

\author{
Ricardo Sandoval' ${ }^{1}$,David Boniface ${ }^{2}$, Randall Stricker ${ }^{3}$, Roman Cregg4 \\ 1. MSC Consultant in Pain Medicine, UCLH and Honorary Clinical Senior Lecturer, Division of Brain Repair and \\ Rehabilitation, Institute of Neurology, UCL. \\ 2.MA, MSc, MBPsS, CStatConsultant in Pain Medicine, UCLH and Honorary Clinical Senior Lecturer, Division of Brain Repair \\ and Rehabilitation, Institute of Neurology, UCL. \\ 3. RN MSc Lead Specialist Pain Nurse UCLHConsultant in Pain Medicine, UCLH and Honorary Clinical Senior Lecturer, \\ Division of Brain Repair and Rehabilitation, Institute of Neurology, UCL. \\ 4. MBBS (Hons), FRCA, FFPMRCA (Exam), PhD (Lond)Consultant in Pain Medicine, UCLH and Honorary \\ Clinical Senior Lecturer, Division of Brain Repair and Rehabilitation, Institute of Neurology, UCL.
}

\section{ARTICLE INFO}

\section{Corresponding Author:}

Ricardo Sandoval

MSC Consultant in Pain Medicine, UCLH and Honorary Clinical Senior Lecturer, Division of Brain Repair and Rehabilitation, Institute of Neurology, UCL.

Keywords:Opioids; $\quad$ circadian rhythms; postoperative pain; patient-controlled analgesia.

\begin{abstract}
Circadian rhythms have governed the everyday life of every single organism that has lived on Earth. The present study addresses the rhythms of cortisol and melatonin, their analgesic properties and the potential circadian rhythm of pain as a driver of the frequency of self-administered analgesia in postoperative patients with an opioid Patient Controlled Analgesia (PCA) delivery system. It aims to determine if acute 24-hour post-operative pain displays a circadian variation by analysing the number of times that patients self-administered morphine for pain relief and incidentally to determine if gender has any association with the frequency of self-administered analgesia. For that purpose, the frequencies of self-administered analgesia were divided into four periods of six hours each (three of them approximately corresponded to the day, and 1 to the night). A Multi-level Poisson regressionanalysis compared frequencies during period 4 (night) to all others $(1,2$ and 3$)$. The results show that there was a statistically significant difference between the frequencies of self-administered opioids in the night period compared to any other day period ( $p$-value of $<0.001$, for periods 1,2 and 3 respectivelycompared to period 4). Differences in terms of gender were also statistically significant $(\mathrm{p}<0.001)$ with men's opioid consumption almost double that of women's but with much steeper rate of decline $(\mathrm{p}<0.001)$. These results may be partly explained by the rhythms of melatonin, cortisol and ß-endorphine, morphine's chronopharmacology and possibly by oestrogen and progesterone.
\end{abstract}

\section{INTRODUCTION}

\section{Circadian rhythm: Overview}

A circadian rhythm refers to a period of approximately 24 hours. The biological master clock, which governs the pace, is located in the suprachiasmatic nuclei (SCN) in the anterior hypothalamus (Reppert and Weaver 2001) and it is driven by light. Light stimulates/suppresses the expression of certain CLOCK genes who will then control basic behaviours such as wakefulness, sleep and temperature (Cajochen et al. 2003).

\section{Circadian rhythm in pain perception}

The idea that pain perception might display a circadian rhythm pattern is not new. Some 
studies date back to 1975 when Glynn and Lloyd (1975) evaluated the potential relationship between the intensity of pain and a specific hour of the day. Their study included patients with cancer pain, herpetic neuralgia, phantom limb pain, atypical facial pain and complex regional pain syndrome (CRPS). The entire sample displayed a circadian pattern with the maximum pain sensitivity registered at 22:00. In another study, Bellamy et al. (1991) analysed 14 patients suffering from rheumatoid arthritis (RA) and found pain to display a circadian variation with the highest registered intensity during the morning around 7:00 - 8:00. These studies show that the pain threshold and its intensity might depend on the pathophysiology of the different kinds of pain. In all cases however, the pain experienced did display a 24-hour rhythm (Glynn and Lloyd, 1975; Bellamy et al 1991; Boscariol et al 2007; Aya et al 2004; Odrcich et al 2006). Cancer pain sufferers appear to experience more breakthrough pain (BTP) during the late morning around 9:45 - 10:15 (Saini et al., 2012). Consistent with these results, Schiessl et al. (2010), evaluated the use of an opioid-PCA for the treatment of BTP in adults with cancer pain. They found that opioid analgesia was least demanded from 22:00 to 6:00 compared to any other time of the day with a significant $34 \%$ less self-administered opioid within these hours.

Inflammatory, autoimmune pain displayed the highest intensity during the early morning at 7:00 (Bellamy et al., 1991). Visceral pain (colic pain) peaks around midnight (Rigas et al., 1990) whereas neuropathic pain increases during the day to reach a peak at 22:00 (Odrcich et al., 2006). Migraine pathophysiology is believed to have a vasogenic origin associated with platelet aggregation and ischaemia which are mechanisms that are also involved in angina pectoris. Interestingly, Solomon, GD (1992) and Willish et al. (2004) arrived to the same conclusion regarding the daytime incidence of these two pain related disorders. Finally, postoperative pain was also worse during the early morning (Boscariol et al., 2007).

The present study aims to determine if acute post-operative pain, indicated by the patients' opioid requirements (by an opioid PCA delivery system), displays a circadian rhythmicity during the first 24 post-operative hours. We hypothesised thatthe pain would display a consistent pattern of variation that is related to the circadian rhythm. We also aimed to identify other variables that could influence this potential rhythm.

\section{Materials and Methods}

The study was an observational prospective study conducted at the University College Hospital London (UCLH). All post-operative patients older than 18 years on an opioid PCA delivery system, including opioid naive patients were included in the study. Patients receiving an epidural or any form of regional analgesia, those with a cancer, haematological, psychiatric diagnosis, chronic pain syndrome and opioid dependent or chronic opioid use were excluded. Also excluded were all incomplete cycles (patients with less than a 24-hour cycle of PCA therapy). A continuous period of 24 hours following surgery was recorded for each patient. The patient must have recovered consciousness and the pain be controlled before he or she is discharged from the recovery ward (RW) The PCA delivery system is installed and ready to be used once the patient leaves the RW. The following data were collected: age, gender, surgical procedure, concomitant administered drugs at the time of the measurement, the type of opioid, the dose, and the time of day and time since surgery at which it was self-administered, the presence or absence of clinician boluses and the PCA status - either PCA-only or PCA + Continuous Infusion. The data collection was performed in London, UK during the months of June and July. The day was defined as the period between 5:00 and 22:59 and night that between 23:00 and 4:59. This timing reflects London's latitude of $51^{\circ} 32^{\prime}$ north where the sun rises at 5:00 and complete darkness occurs at 23:00.

The PCA pumps used at UCH were made by Smiths Medical ASD, Inc. (St. Paul, MN 55112 USA) and are regularly tested for electrical safety. The data were collected every day from each patient's PCA pump at 8:00. All pumps have a standardised protocol of usage, which includes a lockout period of 5 minutes following the administration of an intravenous dose of $1 \mathrm{mg}$ of morphine delivered by the patient. All patients 
were informed about the study and provided oral consent before their data was captured from the PCA pump.

\section{Statistical Analyses}

The mean number of presses each hour were calculated and form the primary outcome measure. To determine if there was a particular period of the day on which the demand for analgesia was more prevalent, the 24-hour day was divided into 4 periods as follows: Period 1 from 5:00 to 10:59, period 2 from 11:00 to 16:59, period 3 from 17:00 to 22:59 and period 4 (night) from 23:00 to 4:59. A generalised linear mixed model with Poisson link regressed frequency of demanded analgesia on period of the day, gender and time since surgery was performed. Potentially confounding variables included the type of operation (degree of invasiveness) and the time at which surgery was performed (morning, afternoon, evening and night)

\section{Results}

In total, data for 16 patients (8 men and 8 women) were included; $18.75 \%$ were between 18 and 30 years old, $37.5 \%$ between 40 and 65 and $43.75 \%$ were older than 65 . All patients used a morphine PCA delivery system, 31.25\% (5 patients) had a continuous infusion, 2 of them at a rate of $0.5 \mathrm{mg} / \mathrm{h}$ and three of them at $1 \mathrm{mg} / \mathrm{h}$; $62 \%$ were also having oral paracetamol for pain relief and $31.25 \%$ were receiving ibuprofen. Other oral medications that were administered during the 24-hour cycle included antibiotics, antihypertensives, anticoagulation and fat lowering therapy. Only 1 patient was on prednisolone. The mean number of presses for all 16 patients is displayed as a bar chart for all 24 hours recorded for each patient (Fig 1). Relatively low frequencies are seen during the night (Fig 2).

In addition to period, time since operation (hr), gender and interactions were included in the model. All main effect terms and interaction period*post-op-hours were statistically significant with $\mathrm{p}<0.001$, interactions of period*gender with $\mathrm{p}=0.028$. Table 1 .

Contrasts of differences between mean presses at period 4 (night time) and periods 1, 2 and 3 show higher day time values and are statistically significant with p-values $<0.001$.
Results for secondary factors were of a strong effect of time since operation, gender and their interaction. These effects are illustrated in Fig 3. The results indicated in Fig 1 and Fig 2, suggest that the alternative hypothesis is true and that there is a circadian rhythm in pain with more incidence during the day compared to time during the night. The trajectory plot, Fig 3, shows the mean frequencies for the demanded analgesia as a function of the postoperative period. It is clear (and was expected) that as time passed, pain reduced. This result suggested a possible confounding effect from the time of the operation. However, including the hour of the day at which the surgery was performed in the statistical model found no noticeable effect. With regard to differences between the frequencies of self-administered doses in terms of gender, there was a statistically significant difference ( $p$-value of $<0.001$ ) Fig 3 with men taking almost double the self-administered opioid analgesia compared to women.

\section{Discussion}

Acute pain as a measure of patient's opioid requirements does display a circadian rhythmicity during the first 24-postoperative hours. It is less prevalent during the night compared to the day. In terms of gender, women self-administered less opioid for pain relief compared to men under the same post-surgical background. The circadian behaviour of pain and analgesia observed herein could be partly explained by the circadian rhythms of analgesic hormones such as cortisol, melatonin and BEndorphine.

\section{Cortisol, melatonin and ßEND and their circadian rhythms on pain and analgesia.}

Human studies have proven the existence of a diurnal cortisol rhythm by measuring cortisol present in saliva of healthy volunteers (Thorn et al. 2004 and Wüst et al. 2000). Debono et al. (2009), studied the circadian variation in serum cortisol in 33 healthy participants demonstrating that the cortisol levels peaked at 08:32 and were lowest at 00:18.If cortisol is influenced by light; is it alsoinfluenced by melatonin? Could melatonin drive the circadian rhythm of cortisol? Can these variables explain the circadian rhythmicity in postoperative pain in this study? 


\section{Melatonin, cortisol and BEND}

Melatonin appears to drive the secretion an important endogenous analgesic: BEND. Barret et al., (1993) performed a study in rats in which they evaluated the role of melatonin as a regulator of the secretion of BEND in rats and whether its secretion was related to analgesia by changes in pain behaviours. The suppression of melatonin secretion was correlated with an increase in the production of endogenous BEND. The high concentrations of BEND were then correlated to an increase in the timelatencies on a hot plate providing proof of an analgesic effect for these rats. The authors concluded that 'there is an inverse phase relationship between melatonin and BEND resulting from a feedback mechanism between the opioid and the pineal system'. It is important to highlight the fact that ßEND and ACTH come from the same macromolecule: the POMC (Proopiomelanocortin), and that the increase in ßEND via POMC would also raise ACTH. ACTH would then promote the secretion of cortisol via adrenals and would alsoprovideanalgesiceffects. Some authors claim that melatonin has an analgesic effect of itself (Lakin et al.1981; Golombek et al. 1990; Chang-Xi Yu et al. 2000). This property would explain the decreased demand for analgesia during the night. If patients suffer less from pain at night and if BEND levels are known to be low at that point, then the explanation of the decrease in pain intensity (and hence of the analgesic requirements) could be attributed to the analgesic properties of increased melatonin serum concentrations at night.

\section{Surgery and its influence on cortisol's and melatonin's circadian rhythms}

Cortisol is the most important stress hormone and its secretion increases rapidly once surgery has started. Most importantly, during the immediate postoperative period both hormones (ACTH and cortisol) remain constantly high (Desborough, 2000). Dimopoulou et al. (2008) found that during PO day 1, cortisol and ACTH levels were both high, while during PO day 2, cortisol levels were still high but in a context of low ACTH. Cortisol was also found by Vogeser et al. (1999), Gogenur et al. (2007), Hendolin et al. (2000) and Ortega et al. (1996) to be increased after surgery.
Gogenur et al. (2007) evaluated alterations in melatonin secretion after major surgery. They measured melatonin in PO patients and found two statistically significant differences in melatonin: Its rhythm was preserved but was shifted to a later time of the night and its concentration augmented from a mean preoperative value of $51 \mathrm{pg} / \mathrm{ml}$ to $68 \mathrm{pg} / \mathrm{ml}$ on PO day 2. The shift in time was present from PO day 1 persisting onto PO day 2 manifested as a delay in melatonin's peak onset from 22:46 to 23:54.

It is therefore reasonable to conclude that the stress of surgery keeps cortisol levels high during at least the first 2 PO days in the body's attempt to deal with the physical stress but also in a way to provide the appropriate setting for repair, recovery and analgesia. Melatonin levels after surgery are also affected; this alteration is initially manifested as a delay on the onset of its rhythm and afterwards as an increase in its concentration. Surgical procedures appear to blunt the circadian pattern of activity of cortisol and melatonin with a markedincrease intheir concentrations compared totheir basal state. See Fig. 4 for summary.

\section{Morphine'schronophysiologyand pharmacology}

Chronopharmacology refers to the study of a drug's pharmacodynamics and pharmacokinetics as a function of time. Apart from the circadian variation in the secretion of endogenous opioids and other hormonesthat influence them, the daytime variability in the expression of opioids' receptors, as well as their bioavailability, can partly account for the rhythmicity of postoperative pain intensities. If morphine is better absorbed and better distributed during the night and if this circumstance coincides with the time at which $\mathrm{Mu}$ opioid receptors are at their highest expression, then the analgesia would be much more efficient during the night compared to the day.

Takada et al. (2013) evaluated the circadian variations in pain sensitivity in mice subjected to a pain model (lesioned mice) compared to control (healthy) mice whilst measuring the potential variations in the expressions of $\mathrm{Mu}$ - 
receptor mRNA in different brain regions (including the periaqueductal grey mater (PAG)). They observed that the pain behaviour was at its highest between 14:00 and 20:00 in the lesionedmice (tested by the hot plate); the same period of time on which the lowest expression of $\mathrm{Mu}$ receptor mRNA in the (PAG) was documented in thesemice.In contrast, control-mice showed a greater latent period in the hot plate between 14:00 and 20:00 which also corresponded to the highest levels of mRNA Mu opioid receptor in the PAG in these healthy mice. Their results show two important facts: Firstly, that pain behaviours can be explained in terms of the availability of $\mathrm{Mu}$ opioid receptors in pain regulating areas. Secondly, there is a rhythm in opioid receptor expression and this rhythm is subject to changes as a consequence of astressfulevent: an invasive lesion or surgery.

\section{Differences in pain perception as a function of gender}

The idea that pain tolerance and pain thresholds are different between men and women is not new and recent studies not only support that idea but also have obtained the same results as the present study.Chia et al (2002) evaluated the morphine requirements in postoperative patients who were on PCA analgesia. They found out that the most important factors influencing the self-administration of morphine was gender (and pain upon movement). With a total of 2298 patients analysed, women consumed significantly less morphine during the first 3 postoperative days compared to men $(23 \%$ to $43 \%$ more morphine consumption in men). McQuay et al. (1980) also arrived to the same results whilst comparing the demanded analgesia in the postoperative period between men and women on an opioid PCA.

Miaskowski and Levine (1999) performed a systematic review to establish if there were any differences in gender relative to postoperative pain and the use of an opioid PCA. Of the 18 studies analysed, 56\% showed more opioid consumption by men in the immediate postoperative period. However, the author also remarks that many of these studies did not measure pain intensity (i.e. with a validated pain scale: VAS) and relied on analgesic consumption to account for these differences. Pain and analgesia are two different things but they risk being mixed together. To highlight this difference is crucial and can be exemplified as follows: If women consume fewer opioids than men while they are in pain, it can be said that opioids are more efficient for pain relief in women as compared to men but it can also be explained by arguing that women suffer less from pain than men.

\section{Conclusions}

The literature as well as the present study points toward a circadian rhythm in pain that might depend on many complex variables that pose a challenge to their quantification such as the drug's variation of its bioavailability, the expression of opioid receptors and their location and the variability in the expression of such receptors as a consequence of invasive procedures. While the circadianrhythms of melatonin and cortisol getdisrupted by surgery, theirhighlevelscannotexplain the diminishedfrequency in selfadministeredanalgesiaat night.

The fact that women self-administered less morphine than men suggests either that opioids work better in this group or that women suffer from less pain than men. Eitherway, the pain isthere and if pain is to be prevented rather than treated, then the way to provide analgesia will need a system capable of delivering the exact amount of dose at its most efficient time while considering the time at which the pain is most probable to occur.

\section{Acknowledgments}

R.Sandoval - CONACyT (ConsejoNacional de Ciencia y Tecnología) Founded Ricardo Sandoval's Masters Degree.

R.C.This project was supported by the National Institute for Health Research University College London Hospitals Biomedical Research Centre.

There are no Conflicts of interest.

\section{References}

1. Aya AG, Vialles N, Mangin R, Robert C, Ferrer JM, Ripart J, et al. Chronobiology of labour pain perception: an observational 
study. British journal of anaesthesia. 2004;93(3):451-3.

2. Bellamy N, Sothern RB, Campbell J, Buchanan WW. Circadian rhythm in pain, stiffness, and manual dexterity in rheumatoid arthritis: relation between discomfort and disability. Annals of the Rheumatic Diseases. 1991;50(4):243-8.

3. Boscariol R, Gilron I, Orr E. Chronobiological characteristics of postoperative pain: diurnal variation of both static and dynamic pain and effects of analgesic therapy. Canadian journal of anaesthesia = Journal canadiend'anesthesie. 2007;54(9):696704.

4. Cajochen C, Krauchi K, Wirz-Justice A. Role of melatonin in the regulation of human circadian rhythms and sleep. Journal of neuroendocrinology. 2003;15(4):432-7.

5. Chia YY, Chow LH, Hung CC, Liu K, Ger LP, Wang PN. Gender and pain upon movement are associated with the requirements for postoperative patientcontrolled iv analgesia: a prospective survey of 2,298 Chinese patients. Canadian journal of anaesthesia $=$ Journal canadiend'anesthesie. 2002;49(3):24955.

6. Debono M, Ghobadi C, Rostami-Hodjegan A, Huatan H, Campbell MJ, Newell-Price J, et al. Modified-release hydrocortisone to provide circadian cortisol profiles. The Journal of clinical endocrinology and metabolism. 2009;94(5):1548-54.

7. Desborough JP. The stress response to trauma and surgery. British journal of anaesthesia. 2000;85(1):109-17.

8. Dimopoulou I, Tzanela M, Vassiliadi D, Mavrou I, Kopterides P, Orfanos S, et al. Pituitary-adrenal responses following major abdominal surgery. Hormones (Athens, Greece). 2008;7(3):237-42.

9. Gasche Y, Daali Y, Fathi M, Chiappe A, Cottini S, Dayer $P$, et al. Codeine

Intoxication Associated
UltrarapidCYP2D6 with
England Journal of $\quad$ Medicine.
2004;351(27):2827-31.

10. Glynn CJ, Lloyd JW. The diurnal variation in perception of pain. Proceedings of the Royal Society of Medicine. 1976;69(5):369-72.

11. Gogenur I, Ocak U, Altunpinar O, Middleton B, Skene DJ, Rosenberg J. Disturbances in melatonin, cortisol and core body temperature rhythms after major surgery. World journal of surgery. 2007;31(2):290-8.

12. Golombek DA, Escolar E, Burin LJ, De Brito Sanchez MG, Cardinali DP. Timedependent melatonin analgesia in mice: inhibition by opiate or benzodiazepine antagonism. European journal of pharmacology. 1991;194(1):25-30.

13. Hendolin HI, Paakonen ME, Alhava EM, Tarvainen R, Kemppinen T, Lahtinen P. Laparoscopic or open cholecystectomy: a prospective randomised trial to compare postoperative pain, pulmonary function, and stress response. The European journal of surgery $=$ Actachirurgica. 2000;166(5):394-9.

14. Ishida A, Mutoh $T$, Ueyama $T$, Bando $H$, Masubuchi S, Nakahara D, et al. Light activates the adrenal gland: timing of gene expression and glucocorticoid release. Cell metabolism. 2005;2(5):297-307.

15. Lakin ML, Miller CH, Stott ML, Winters WD. Involvement of the pineal gland and melatonin in murine analgesia. Life sciences. 1981;29(24):2543-51.

16. Landau R. Polymorphisme génétique et traitement par les opiacés. La Presse Médicale. 2008;37(10):1415-22.

17. Marc LCitron ,Jagmohan Kalra , Vicki L Seltzer, Sidney Chen, Mark Hoffman, Mary B. Walczak. PatientControlledAnalgesia for Cancer Pain: A Long-TermStudy of Inpatient and Outpatient Use.Cancer Investigation Vol. 
10, Iss. 5, 1992.

18. McQuay HJ, Bullingham RE, Paterson GM, Moore RA. Clinical effects of buprenorphine during and after operation. British journal of anaesthesia. 1980;52(10):1013-9.

19. Miaskowski C, Levine JD. Does opioid analgesia show a gender preference for females? Pain Forum. 1999;8(1):34-44.

20. Niijima A, Nagai K, Nagai $N$, Nakagawa $H$. Light enhances sympathetic and suppresses vagal outflows and lesions including the suprachiasmatic nucleus eliminate these changes in rats. Journal of the Autonomic Nervous System. 1992;40(2):155-60.

21. Odrcich M, Bailey JM, Cahill CM, Gilron I. Chronobiological characteristics of painful diabetic neuropathy and postherpetic neuralgia: diurnal pain variation and effects of analgesic therapy. Pain. 2006;120(1-2):207-12.

22. Ortega AE, Peters JH, Incarbone R, Estrada L, Ehsan A, Kwan Y, et al. A prospective randomized comparison of the metabolic and stress hormonal responses of laparoscopic and open cholecystectomy. Journal of the American College of Surgeons. 1996;183(3):249-56.

23. Petraglia F, Facchinetti F, Parrini D, Micieli G, De Luca S, Genazzani AR. Simultaneous Orcadian Variations of Plasma ACTH, Beta-Lipotropin, BetaEndorphin and Cortisol. Hormone Research in Paediatrics. 1983;17(3):14752.

24. Reppert SM, Weaver DR. Molecular analysis of mammalian circadian rhythms. Annual review of physiology. 2001;63:647-76.

25. Rigas B, Torosis J, McDougall CJ, Vener KJ, Spiro HM. The circadian rhythm of biliary colic. Journal of clinical gastroenterology. 1990;12(4):409-14.

26. Saini A, Tucci M, Tampellini M, Maina D, Bouraouia K, Giuliano PL, et al. Circadian variation of breakthrough pain in cancer patients. European journal of pain (London, England). 2013;17(2):264-70.

27. Schiessl C, Schestag I, Sittl R, Drake R, Zernikow B. Rhythmic pattern of PCA opioid demand in adults with cancer pain. European journal of pain (London, England). 2010;14(4):372-9.

28. Solomon GD. Circadian rhythms and migraine. Cleveland Clinic journal of medicine. 1992;59(3):326-9.

29. Takada T, Yamashita A, Date A, Yanase M, Suhara Y, Hamada A, et al. Changes in the circadian rhythm of mRNA expression for micro-opioid receptors in the periaqueductal gray under a neuropathic pain-like state. Synapse (New York, NY). 2013;67(5):216-23.

30. Thorn L, Hucklebridge F, Esgate A, Evans $\mathrm{P}$, Clow A. The effect of dawn simulation on the cortisol response to awakening in healthy Psychoneuroendocrinology. 2004;29(7):925-30.

31. Tracey Barrett, Stephen Kent, Nicholas Voudouris, Doesmelatoninmodulatebetaendorphin, corticosterone, and pain threshold?, Life Sciences, Volume 66, Issue 6, 2000, Pages 467-476, ISSN 00243205, http://dx.doi.org/10.1016/S00243205(99)00616-5.

32. Vogeser M, Felbinger TW, Kilger E, Roll W, Fraunberger P, Jacob K. Corticosteroidbinding globulin and free cortisol in the early postoperative period after cardiac surgery. Clinical biochemistry. 1999;32(3):213-6.

33. Weibel L, Brandenberger G. The start of the quiescent period of cortisol remains phase locked to the melatonin onset despite circadian phase alterations in humans working the night schedule. Neuroscience letters. 2002;318(2):89-92.

34. Willich SN, Kulig M, Muller-Nordhorn J. European survey on circadian variation of angina pectoris (ESCVA) in treated patients. Herz. 2004;29(7):665-72. 
Circadian variation of pain as a measure of the analgesia requirements during the first 24-postoperative hours in patients using an opioid Patient Controlled Analgesia delivery system

35. Wust S, Wolf J, Hellhammer DH, Federenko I, Schommer N, Kirschbaum C. The cortisol awakening response - normal values and confounds. Noise \& health. 2000;2(7):79-88.

36. Yu CX, Wu GC, Xu SF, Chen CH. Effect of melatonin on release of beta-endorphin, norepinephrine and 5-hydroxytryptamine in rat brain. Yao xuexuebao = ActapharmaceuticaSinica. 2001;36(1):5-9.

\section{Figure Legends:}

Fig 1. Mean frequency for every hour (pressed button/hour). In yellow is depicted the day-hours and in blue the night-hours.

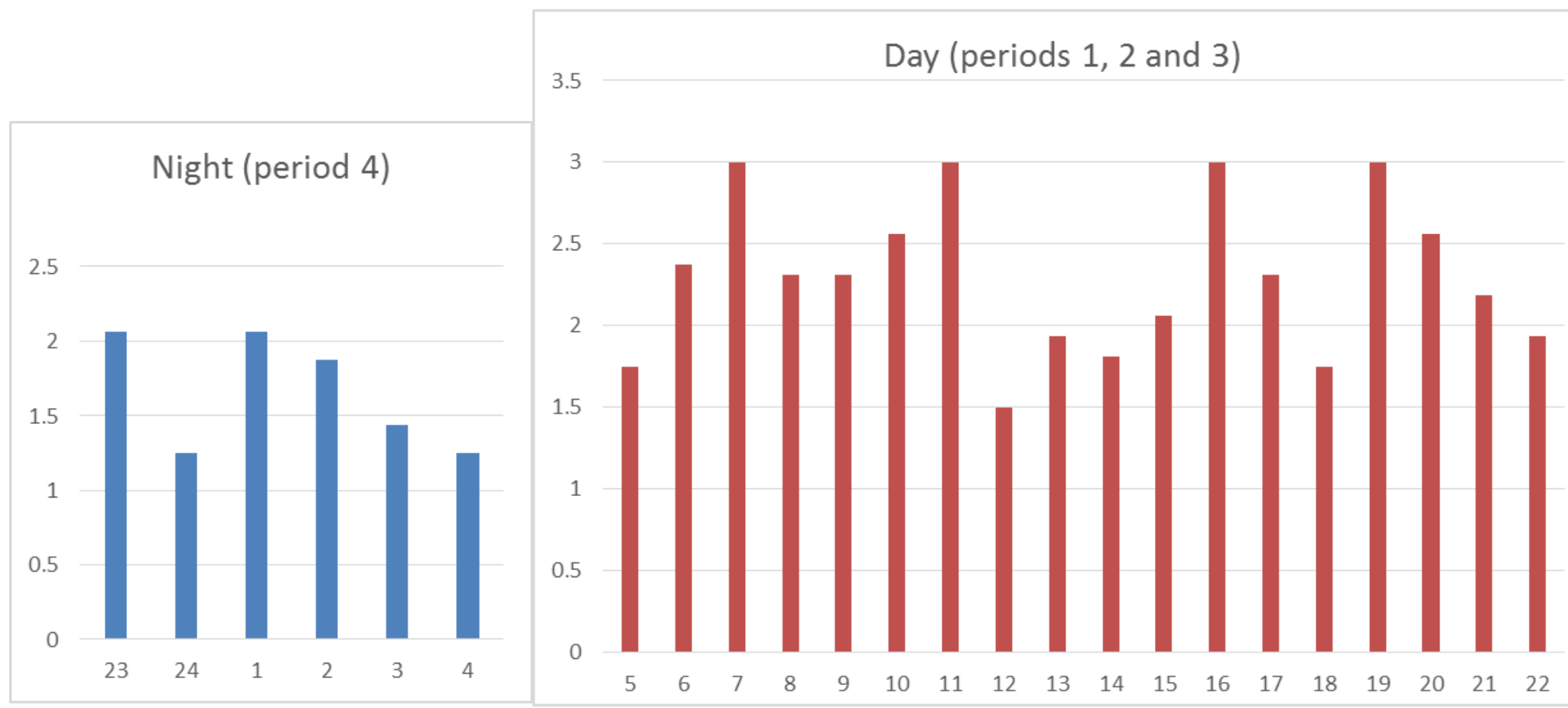

Fig 2. Mean rate of administrations of pain relief for eachperiod: Period 1: 05:00 - 10:59, period 2: 11:00 - 16:59, period 3:17:00 - 22:59 and period 4 (night): 03:00 - 4:49.

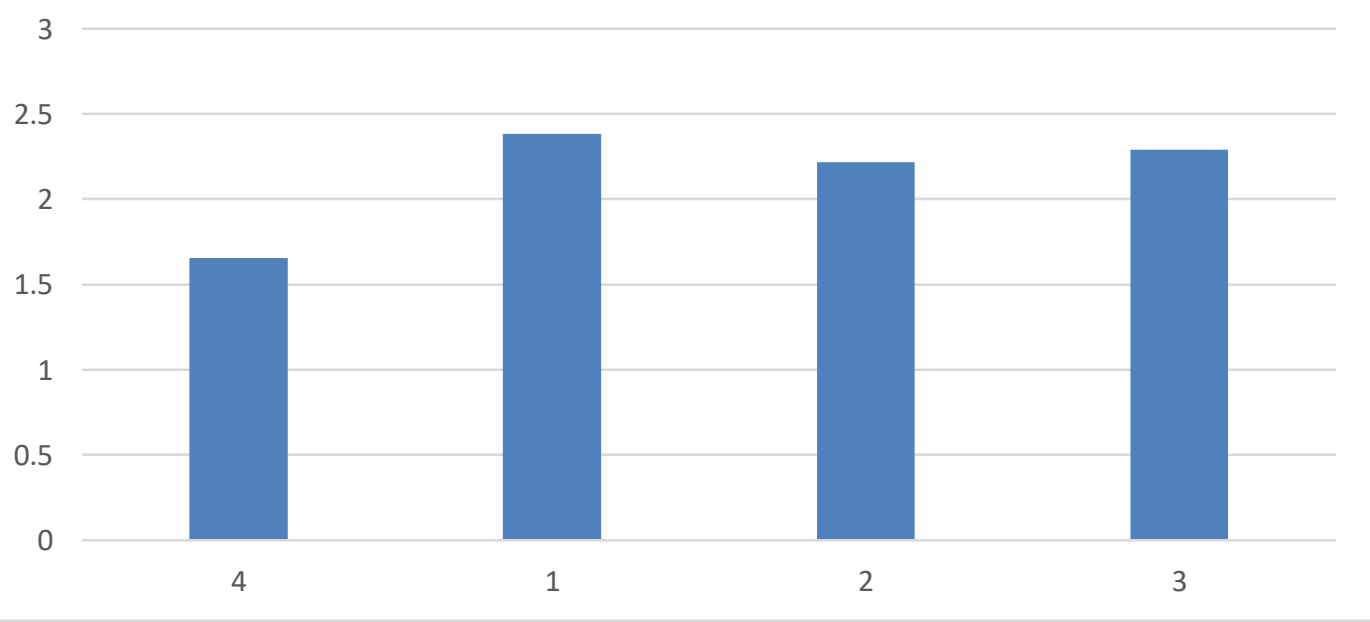


Circadian variation of pain as a measure of the analgesia requirements during the first 24-postoperative hours in patients using an opioid Patient Controlled Analgesia delivery system

Fig 3.Trajectories of pressing rate by post-op hours for 8 males and 8 females (from fitted statistical model).

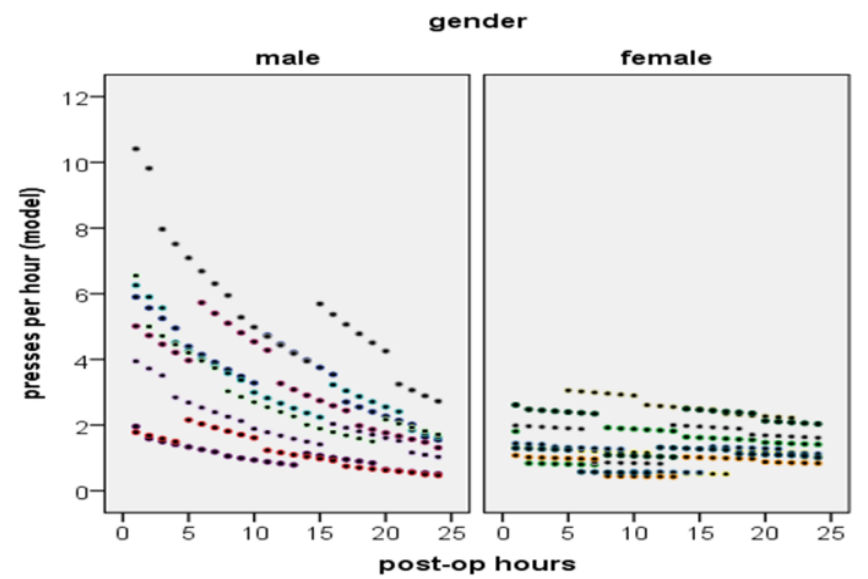

Fig 4.Summary of how light, darkness and surgery affect the circadianrhythm of cortisol and melatonin.

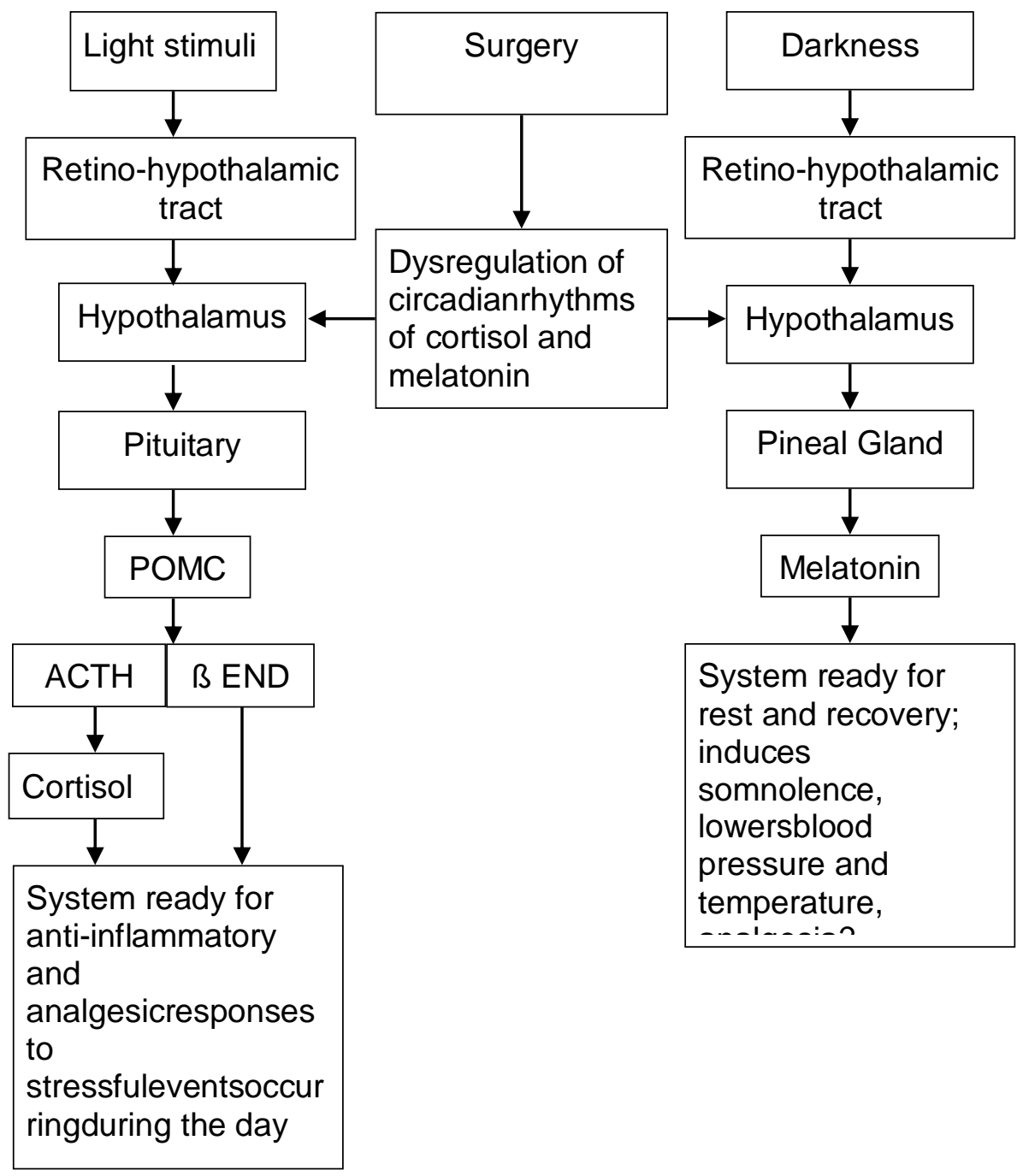


Circadian variation of pain as a measure of the analgesia requirements during the first 24-postoperative hours in patients using an opioid Patient Controlled Analgesia delivery system

Table 1. Significance tests of fixed effects terms in the statistical model for 'times pressed button' (using Poisson distribution and log link function)

\begin{tabular}{|l|c|r|r|r|}
\hline Source & F & df1 & df2 & p-value \\
\hline \hline Corrected Model & 11.511 & 9 & 374 & 0.001 \\
\hline Period & 10.300 & 3 & 374 & 0.001 \\
\hline Gender & 16.455 & 1 & 374 & 0.001 \\
\hline Period*gender & 3.073 & 3 & 374 & 0.028 \\
\hline Post_op_hrs & 35.278 & 1 & 374 & 0.001 \\
\hline Gender*post_op_hrs & 16.584 & 1 & 374 & 0.001 \\
\hline
\end{tabular}

